\title{
CURSOS DIURNOS E NOTURNOS: FATORES DE APROVAÇÃO NO VESTIBULAR DA UFMG
}

\author{
TALITA F. S. DIAS \\ LUDMILA V. LAGE \\ RAPHAEL L. F. RIBEIRO \\ GUSTAVO H. M. A. ROCHA \\ JUNIO G. RODRIGUES \\ THIAGO R. SANTOS \\ GLAURA C. FRANCO \\ ROSANGELA H. LOSCHI \\ MAURO M. BRAGA
}
Departamento de Estatística do Instituto de Ciências
Exatas da Universidade Federal de Minas Gerais
dest@est.ufmg.br

\begin{abstract}
RESUMO
Esse estudo analisa dados do vestibular da Universidade Federal de Minas Gerais de 2004, mediante um modelo de regressão não paramétrico, o Classification and Regression Trees. Seu objetivo foi identificar os principais fatores de aprovação e, também, verificar se esses fatores eram os mesmos para os cursos diurnos e noturnos. A resposta a essas questões permitiria verificar se a expansão do turno noturno feita por essa universidade vinha promovendo maior inserção social. Observou-se que, em geral, a conclusão do ensino médio em escolas públicas federais ou particulares, o conhecimento de língua estrangeira e o pertencimento a um grupo socioeconômico alto são fatores fortemente associados à aprovação do candidato. Verificou-se, ainda, que nos cursos noturnos as variáveis socioeconômicas têm maior relevância, enquanto nos cursos diurnos a formação do candidato adquire maior peso. Finalmente, o fator socioeconômico médio tende a ser maior para os candidatos aprovados.

RAÇA - CONDIÇÕES SOCIOECONÔMICAS - RENDIMENTO ESCOLAR - UNIVERSIDADE
\end{abstract}

Agradecemos aos professores Antônio de Araújo, Maria do Carmo L. Peixoto e Antônio Zumpano P. Santos, da Universidade Federal de Minas Gerais - UFMG -, pelos comentários e sugestões; à Comissão Permanente do Vestibular - Copeve - da UFMG, por ceder o banco de dados e ter contribuído com o financiamento dessa pesquisa. Rosangela $\mathrm{H}$. Loschi também contou com o apoio parcial do Conselho Nacional de Desenvolvimento Científico e Tecnológico - CNPq - e Glaura Franco foi apoiada, em parte, pelo CNPq e, em parte pela Fundação de Amparo à Pesquisa no Estado de Minas Gerais - Fapemig. 
Talita F. S. Dias, Ludmila V. Lage, Raphael L. F. Ribeiro, Gustavo H. M. A. Rocha, Junio G. Rodrigues, Thiago R. Santos, Glaura C. Franco, Rosangela H. Loschi e Mauro M. Braga

\begin{abstract}
DAYTIME AND NIGHT TIME COURSES: FACTORS THAT LED TO APPROVAL IN 2004 ENTRANCE EXAMINATION FROM FEDERAL UNIVERSITY OF MINAS GERAIS. This study analyses data from 2004 Federal University of Minas Gerais' general entrance examination, making use of a non-parametrical regression model: the Classification and Regression Trees. Its aims were to identify the main factors of college approval and, also, to verify if these factors were the same for both daytime and nighttime courses, in order to be able to affirm that the expansion of nighttime courses was promoting, in this university, a higher social insertion. In general, it was observed that the attendance to a federal or private high school, the knowledge of foreign languages and a higher socioeconomic status were factors strongly associated with candidates' approval. In nighttime courses, it was found that socioeconomics variables had a higher importance, while variables related to the quality of previous schooling had more weight in daytime courses. The average socioeconomic factor tended to be higher in the group of the approved candidates.

RACE - SOCIOECONOMIC FACTOR - STUDENT ACHIEVEMENT - UNIVERSITY
\end{abstract}

O tema da equidade do acesso ao ensino superior público no Brasil é cada vez mais atual. A forte seletividade social associada aos exames de acesso à universidade pública tem acarretado a adoção de alternativas diferenciadas para enfrentar o problema. A Universidade de São Paulo - USP -, por exemplo, criou curso preparatório gratuito destinado aos estudantes da escola pública e abriu nova unidade na zona leste da cidade de São Paulo. A Universidade Estadual de Campinas - Unicamp - concedeu pontos adicionais aos estudantes das escolas públicas e aos que se declaram indígenas, pretos ou pardos. A Universidade Federal de Pernambuco - UFPE - adotou, no caso das licenciaturas da área de exatas, um processo de seleção que envolve o oferecimento, por parte da universidade de um curso preparatório, gratuito, para a segunda etapa do concurso. A Universidade Federal de Minas Gerais - UFMG definiu a expansão no turno da noite como mecanismo prioritário para a inclusão social.

Entre todos os modelos praticados, a alternativa mais usual, e também a que desperta maior polêmica, é a adoção de reserva de vagas para estudantes oriundos de escolas públicas e/ou pertencentes às minorias raciais. Empre-

I. No Rio, a Universidade do Estado do Rio de Janeiro - Uerj - e a Universidade Estadual do Norte Fluminense - Uenf - e, na Bahia, a Universidade do Estado da Bahia - Uneb. 
gado inicialmente pelas universidades estaduais do Rio de Janeiro e da Bahia', esse modelo, com características específicas em cada caso, foi também adotado por diversas instituições federais, tais como a Universidade Federal do Paraná - UFPR - e a Universidade de Brasília - UnB. Os grupos sociais defensores das políticas de ações afirmativas são francamente a favor dessa opção e têm patrocinado campanhas em prol de seu emprego por força de lei. Projetos nesse sentido tramitam no Congresso Nacional e o tema está, inclusive, contemplado na proposta de reforma universitária do governo federal (Informativo MEC, nov. de 2004 e jul. 2005, por exemplo).

A implementação da política de cotas nas universidades brasileiras vem suscitando acirrados debates e, como conseqüência, diversas abordagens sobre o tema têm aparecido na literatura atual. Entre elas, podemos citar os trabalhos de Guimarães (2003), Pereira (2003), Azevedo (2004), Gomes (2004), Maggie e Fry (2004), Peixoto e Braga (2004), Lage et al. (2007) e Lopes et al. (2007).

Este trabalho visa, a partir da análise estatística dos dados do vestibular da UFMG de 2004, avaliar a pertinência da decisão da UFMG de adotar a expansão de vagas no turno noturno como alternativa preferencial para a inclusão social. Desta forma, pretendemos identificar os principais fatores que levaram o candidato à aprovação considerando a totalidade dos candidatos inscritos no vestibular-2004 e os inscritos para os cursos diurnos e noturnos, verificando se há diferença nos fatores que levam à aprovação em cada um desses grupos.

O trabalho está organizado da seguinte forma: na segunda seção descrevemos as variáveis utilizadas e fazemos uma breve descrição da metodologia; na terceira seção apresentamos algumas características dos cursos diurnos e noturnos; na quarta seção utilizamos o Classification Regression Trees [Árvores de Classificação e Regressão] - Cart - para identificar as variáveis mais associadas à aprovação e fazemos uma descrição dos nós com mais alta e mais baixa probabilidade de aprovação; na quinta seção apresentamos uma análise dos candidatos em relação ao fator socioeconômico; a sexta seção finaliza o artigo apresentando as conclusões mais relevantes.

\section{ASPECTOS METODOLÓGICOS}

trabalho está centrado na análise das informações obtidas no ques- 
Talita F. S. Dias, Ludmila V. Lage, Raphael L. F. Ribeiro, Gustavo H. M. A. Rocha, Junio G. Rodrigues, Thiago R. Santos, Glaura C. Franco, Rosangela H. Loschi e Mauro M. Braga

tionário socioeconômico respondido pelos candidatos ao vestibular 2004 da UFMG quando se inscreveram no concurso, confrontando-se as respostas dos estudantes aprovados nesse certame com a dos que não o foram. As variáveis do questionário socioeconômico da UFMG podem ser divididas basicamente em seis tipos:

\section{Variável resposta:}

candidato foi aprovado?

\section{Variáveis explicativas:}

I. Variáveis socioeconômicas:

Trabalha em atividade remunerada?

Por quantos anos teve atividade remunerada?

Renda familiar

Participação na vida econômica do grupo familiar

Número de pessoas que vivem da renda mensal

Classificação social Abipeme ${ }^{2}$

Classificação social fator socioeconômico

2. Variáveis relativas à formação do candidato no segundo grau:

Ano de conclusão do ensino médio

Onde conclui o ensino médio

Tipo de ensino médio

Tipo de escola de ensino médio

Curso de ensino médio

Quantos anos levou para concluir o ensino médio

Turno do ensino médio

3. Variáveis relativas ao histórico do candidato em vestibulares:

Há quanto tempo tenta ingressar em curso superior

Já prestou algum vestibular

Quantos vestibulares já prestou

Freqüentou cursinho pré-vestibular

Motivo da escolha do curso

2. Associação Brasileira de Institutos de Pesquisa de Mercado. 
Cursos diurnos e noturnos...

4. Variáveis relativas ao conhecimento de língua estrangeira:

Freqüentou cursinho de idiomas

Situação com respeito à língua estrangeira

Opção de língua estrangeira ${ }^{3}$

5. Variáveis relativas aos pais:

Escolaridade do pai

Escolaridade da mãe

Situação familiar

Ocupação do pai

Ocupação da mãe

6. Variáveis relativas às características pessoais do candidato:

Sexo

Estado civil

É canhoto?

Onde nasceu

Raça/cor

Onde reside

Sabe usar o computador?

Tipo de residência

Para medir o nível socioeconômico do candidato foi utilizado o índice FSE (fator socioeconômico) apresentado em Braga, Peixoto e Bogutchi (200l) e Araújo et al. (2004). O FSE pode assumir valores de I a I0, sendo que o menor valor está associado à menor classe socioeconômica.

Para a identificação dos fatores que levam à aprovação no vestibular será empregada a técnica de análise estatística conhecida como Cart, considerado como um modelo de regressão não paramétrico que tem por objetivo estabelecer uma relação entre um conjunto de variáveis explicativas e uma única variável resposta, que neste caso é "O candidato foi aprovado?". Ele é ajusta-

3. "Situação com respeito à língua estrangeira" denota o conhecimento do candidato em línguas e "Opção de língua estrangeira" é a escolha de idioma segundo o qual o candidato quer ser avaliado no vestibular. 
Talita F. S. Dias, Ludmila V. Lage, Raphael L. F. Ribeiro, Gustavo H. M. A. Rocha, Junio G. Rodrigues, Thiago R. Santos, Glaura C. Franco, Rosangela H. Loschi e Mauro M. Braga

do mediante sucessivas divisões binárias no conjunto de dados, de modo a tornar os grupos resultantes cada vez mais homogêneos em relação à variável resposta. Quando a variável resposta é dicotômica, que é o caso estudado neste trabalho, além das variáveis explicativas associadas à variável resposta, também se pode obter a probabilidade condicional de a variável resposta resultar em sucesso em cada grupo. Conseqüentemente, para o caso em estudo, uma análise por meio do Cart fornece a probabilidade de aprovação dos candidatos que possuem as características apresentadas em cada grupo resultante da divisão, além das variáveis que melhor explicam a aprovação do candidato. Utilizamos o software estatístico SPSS para realizar a implementação do algoritmo Cart. Para maiores detalhes ver, por exemplo, Bell (1996) e Diniz e Louzada-Neto (2000). Uma vantagem do Cart é que as interações entre as variáveis explicativas são automaticamente captadas pelo modelo. No Cart, as variáveis que fazem parte do modelo não são informadas a priori, diferentemente do que ocorre com os modelos lineares. Além disto, é um modelo de fácil interpretação.

Nos estudos realizados via Cart são feitas duas abordagens. A primeira consiste em analisar os dados para os candidatos em geral, para os candidatos aos cursos diurnos e para os candidatos aos cursos noturnos. A segunda consiste em fazer a análise para os candidatos divididos pela área do curso pretendido. Um outro interesse será determinar o fator socioeconômico - FSE médio dos candidatos e identificar quais cursos apresentam menores médias de FSE. Essa comparação permitirá observar se os cursos noturnos realmente estão atraindo candidatos de classe social mais baixa. Para as análises foram considerados o curso do candidato, a área e também a raça/cor.

\section{ALGUMAS CARACTERÍSTICAS DOS CURSOS DIURNO E NOTURNO}

Visando conhecer um pouco melhor o perfil dos candidatos aos cursos diurnos e noturnos, apresentamos, a seguir, a sua distribuição em cada grupo segundo algumas variáveis julgadas relevantes para o estudo.

Podemos perceber das tabelas I, 2 e 3 que, em 2004, os candidatos aos cursos noturnos em sua maioria possuíam FSE inferior ou igual a 2 e freqüentaram escolas públicas estaduais, além de constituírem o grupo em que se observou o maior percentual de candidatos da raça/cor preta e parda. Já para os candidatos aos cursos diurnos, notamos que apenas 19,19\% deles possuem 
TABELA I

DISTRIBUIÇÃO DOS CANDIDATOS INSCRITOS E APROVADOS SEGUNDO O FATOR SOCIOECONÔMICO

\begin{tabular}{cccc}
\hline FSE & Geral(\%) & Diurno(\%) & Noturno(\%) \\
\hline 0 & $2,00(1,20)$ & $1,50(0,56)$ & $5,99(3,57)$ \\
1 & $8,90(3,70)$ & $6,19(2,06)$ & $21,53(10,02)$ \\
2 & $13,60(6,80)$ & $11,50(5,24)$ & $23,05(13,02)$ \\
3 & $16,70(11,20)$ & $16,70(10,30)$ & $16,61(14,86)$ \\
4 & $11,80(10,20)$ & $12,37(9,78)$ & $9,27(11,98)$ \\
5 & $10,30(9,70)$ & $11,10(9,57)$ & $6,29(10,02)$ \\
6 & $9,30(10,60)$ & $10,10(11,22)$ & $5,22(8,06)$ \\
7 & $8,50(11,30)$ & $9,50(12,33)$ & $3,85(7,25)$ \\
8 & $8,30(12,90)$ & $9,25(13,89)$ & $3,92(8,90)$ \\
9 & $6,50(13,30)$ & $7,22(14,69)$ & $2,80(7,72)$ \\
10 & $4,00(9,20)$ & $4,57(10,36)$ & $1,47(4,60)$ \\
\hline
\end{tabular}

Percentual referente aos candidatos aprovados.

TABELA 2

DISTRIBUIÇÃO DOS CANDIDATOS INSCRITOS E APROVADOS SEGUNDO O TIPO DE ESCOLA DE ENSINO MÉDIO

\begin{tabular}{lccc}
\hline Tipo de escola no ensino médio & Geral(\%) & Diurno(\%) & Noturno(\%) \\
\hline Escola pública federal & $4,60(11,30)$ & $4,24(10,59)$ & $6,27(13,83)$ \\
Escola pública estadual & $40,60(19,90)$ & $38,03(16,75)$ & $52,11(32,05)$ \\
Escola pública municipal & $8,20(5,50)$ & $6,93(4,32)$ & $13,90(9,88)$ \\
Escola particular & $46,30(63,20)$ & $50,50(68,16)$ & $27,02(44,13)$ \\
Curso livre & $0,4(0,20)$ & $0,30(0,18)$ & $0,70(0,11)$ \\
\hline
\end{tabular}

Percentual referente aos candidatos aprovados.

TABELA 3

DISTRIBUIÇÃO DOS CANDIDATOS INSCRITOS

E APROVADOS SEGUNDO A RAÇA/COR

\begin{tabular}{|c|c|c|c|}
\hline Raça/cor & Geral(\%) & Diurno(\%) & Noturno(\%) \\
\hline Branca & $60,20(67,70)$ & $62,10(69,57)$ & $51,42(60,35)$ \\
\hline Preta & $6,50(3,50)$ & $5,71 \quad(2,66)$ & $10,18(6,72)$ \\
\hline Parda & $25,20(21,20)$ & $24,21(20,29)$ & $29,76(24,78)$ \\
\hline Amarela & $2,70(1,50)$ & $2,74(1,46)$ & $2,68(1,65)$ \\
\hline Indígena & $0,60(0,30)$ & $0,54(0,29)$ & $0,62(0,55)$ \\
\hline Não deseja declarar & $4,80(5,80)$ & $4,70(5,73)$ & $5,34(5,95)$ \\
\hline
\end{tabular}

Percentual referente aos candidatos aprovados. 
Talita F. S. Dias, Ludmila V. Lage, Raphael L. F. Ribeiro, Gustavo H. M. A. Rocha, Junio G. Rodrigues,

Thiago R. Santos, Glaura C. Franco, Rosangela H. Loschi e Mauro M. Braga

FSE inferior ou igual a 2, a maioria fez ensino médio em escolas particulares e há predominância de candidatos da raça/cor branca. Um outro fato a ser ressaltado é que, entre as escolas públicas, a probabilidade de aprovação de um candidato proveniente de escola pública federal é bem maior do que a dos candidatos provenientes de escola pública municipal e estadual, sendo esta última a que apresenta as menores chances. Também é possível observar que, tanto para os cursos noturnos quanto para os cursos diurnos, o percentual de candidatos aprovados da raça/cor branca é maior que o percentual de inscritos, em cada grupo.

Verificou-se que a maioria dos candidatos, tanto para os cursos noturnos quanto diurnos, mora em Belo Horizonte, Minas Gerais. No entanto, os cursos noturnos atraem menos pessoas de fora da região de Belo Horizonte $\mathrm{BH}$ - e Grande $\mathrm{BH}$.

\section{CONSTRUÇÃO DO MODELO VIA CART}

Nesta seção são feitas duas análises. A primeira consiste em três árvores de decisão, a saber: "análise para os candidatos em geral", "análise para os candidatos aos cursos diurnos" e "análise para os candidatos aos cursos noturnos". A segunda consiste em oito árvores de decisão feitas para os candidatos divididos pela área do curso pretendido: Ciências Agrárias, Engenharias, Ciências Sociais Aplicadas, Ciências Exatas, Lingüística, Letras e Artes, Ciências Biológicas, Ciências da Saúde e Ciências Humanas, cujos diagramas correspondentes podem ser encontrados em Rodrigues et al., 2005. Os cursos que constituem cada área são exibidos na tabela 5.

\section{Análise das árvores para cada grupo}

Objetivando exemplificar as conclusões obtidas a partir de uma árvore, foram analisados apenas os nós terminais com mais alta e mais baixa probabilidade de aprovação. A chance de aprovação de um candidato qualquer é de 6,7\%. Em uma abordagem mais detalhada, todos os nós terminais deveriam ser analisados. Em cada nó há a probabilidade de aprovação do candidato, caso todos os eventos que antecederam tal nó ocorram. 
Para o conjunto dos candidatos (árvore não mostrada) os que têm menores chances de aprovação ( I ,54\%) são os que nunca prestaram vestibular e não possuem muito conhecimento de línguas estrangeiras. Os candidatos com maiores chances de aprovação (36,36\%) têm algum conhecimento de línguas estrangeiras, moram em $\mathrm{BH}$, cursaram todo o ensino médio em escola pública federal, a mãe possui, pelo menos, o curso superior completo e prestaram anteriormente algum vestibular.

Para os candidatos aos cursos diurnos (Anexo I), a conclusão é idêntica à obtida para o conjunto dos candidatos. A menor probabilidade de aprovação também é de 1,54\%, para o mesmo tipo de candidato. Já para os candidatos com mais alta chance de aprovação (39,47\%) observamos apenas algumas mudanças no perfil em comparação com o conjunto. São aqueles que têm algum conhecimento de línguas estrangeiras, moram em $\mathrm{BH}$, fizeram o ensino médio em escola pública federal, a mãe possui curso superior completo ou mais, já prestaram pelo menos um vestibular e levaram três anos para concluir o ensino médio.

O cenário em relação ao curso noturno é também similar (Anexo 2), mas, verificamos alterações de maior monta no perfil dos candidatos que têm elevadas chances de aprovação. Os com menor chance de aprovação ( I,55\%) têm exatamente o mesmo perfil observado para os outros dois conjuntos. Já os candidatos com mais alta chance de aprovação $(32,57 \%)$ têm algum conhecimento de línguas estrangeiras, moram em $\mathrm{BH}$, possuem FSE maior que 3, fizeram o ensino médio integralmente na rede privada ou integralmente na rede pública, já prestaram vestibular, tiveram atividade remunerada por até um ano ou nunca trabalharam e freqüentaram cursinho pré-vestibular.

Percebemos que, embora não sejam os únicos fatores que determinam a aprovação, não possuir muito conhecimento de línguas estrangeiras e nunca ter prestado vestibular, em geral, diminui a probabilidade de aprovação. Candidatos que têm algum conhecimento de línguas estrangeiras, moram em $\mathrm{BH}$ e que fizeram o ensino médio em escola pública federal, em geral, tendem a ter chance mais alta de aprovação.

Verificamos também algumas diferenças entre os candidatos para os cursos diurnos e noturnos. Nos cursos noturnos, há maior relevância das variáveis socioeconômicas, enquanto, nos cursos diurnos, maior importância de variáveis relativas à formação do candidato. No entanto, isso não significa di- 
Talita F. S. Dias, Ludmila V. Lage, Raphael L. F. Ribeiro, Gustavo H. M. A. Rocha, Junio G. Rodrigues, Thiago R. Santos, Glaura C. Franco, Rosangela H. Loschi e Mauro M. Braga

zer que os candidatos aprovados para o turno da noite têm situação socioeconômica melhor do que a dos aprovados para o curso diurno. Na verdade, ocorre exatamente o contrário, como pode ser visto no que segue.

Em relação à análise feita para os candidatos de acordo com a área do curso pretendido, cujas árvores não foram mostradas, a variável mais associada à aprovação é o FSE, sendo que um valor mais alto do FSE está associado à maior chance de aprovação. O FSE só não é a variável mais fortemente associada à aprovação na área de Saúde, em que há preponderância do local de moradia; na área de Exatas, em que predomina o tipo de escola média freqüentada, e na área de Agrárias, na qual a variável mais importante é a habilidade de leitura em língua estrangeira.

\section{Perfil dos candidatos de alguns grupos específicos}

Analisando o perfil dos candidatos que formam os três nós com probabilidade de aprovação mais alta e os três nós com probabilidade de aprovação mais baixa, para a totalidade dos candidatos e para os candidatos dos cursos noturnos e diurnos, algumas informações importantes são obtidas. Na apresentação dessas informações, utilizaremos a notação apresentada na tabela 4, segundo a qual os nós com mais alta probabilidade de aprovação correspondem a um percentual relativamente baixo da população.

TABELA 4

PROBABILIDADE DE APROVAÇÃO E PERCENTUAL DE CANDIDATOS (NÓS) COM BAIXA E ALTA CHANCE DE APROVAÇÃO

\begin{tabular}{|c|c|c|c|c|c|c|c|}
\hline \multirow{3}{*}{\multicolumn{2}{|c|}{ Chance de Aprovação Nó }} & \multicolumn{6}{|c|}{ Grupo de Candidatos } \\
\hline & & \multicolumn{2}{|c|}{ Geral } & \multicolumn{2}{|c|}{ Diurno } & \multicolumn{2}{|c|}{ Noturno } \\
\hline & & chance & $\%$ & chance & $\%$ & chance & $\%$ \\
\hline \multirow[t]{3}{*}{ Baixa } & I & $\mathrm{I}, 54$ & 21,15 & 1,54 & 21,09 & 1,55 & 21,63 \\
\hline & 2 & 2,59 & 7,14 & 2,62 & 8,42 & 2,90 & 0,64 \\
\hline & 3 & 3,07 & 2,53 & 2,98 & 2,49 & 3,75 & 20,89 \\
\hline \multirow[t]{3}{*}{ Alta } & 4 & 22,75 & 5,52 & 22,80 & 5,61 & 28,21 & 0,73 \\
\hline & 5 & 34,05 & 0,54 & 36,73 & 0,49 & 28,57 & 0,91 \\
\hline & 6 & 36,36 & 0,31 & 39,47 & 0,23 & 32,57 & 4,49 \\
\hline
\end{tabular}


Cursos diurnos e noturnos...

Os nós com probabilidade mais baixa de aprovação (nós I, 2 e 3) são, predominantemente, constituídos por candidatos que cursaram o ensino médio em escolas públicas estaduais, com exceção dos cursos diurnos em dois nós (nós I e 2), em que o percentual de candidatos vindos de escolas particulares e públicas estaduais é bem próximo. Com respeito ao FSE observamos que os candidatos aos cursos noturnos têm, em sua maioria, FSE menor ou igual a 5 e os candidatos aos cursos diurnos têm valor de FSE entre I e 8, predominantemente.

Os nós com maior chance de aprovação (nós 4, 5 e 6) são formados essencialmente por candidatos que fizeram o ensino médio em escolas particulares ou públicas federais, tanto para os cursos noturnos quanto diurnos. Quanto ao nível socioeconômico, os candidatos dos nós com mais alta chance de aprovação tendem a ter um FSE superior ou igual a 5, exceto para os candidatos aos cursos noturnos dos nós 4 e 5, que em sua maioria apresentam FSE menor que 4.

Com respeito à raça/cor percebemos que, embora haja uma concentração maior de candidatos da raça/cor branca em todos os nós, a proporção de brancos é muito maior que as demais para os nós com mais alta chance de aprovação, sendo que essa diferença é mais acentuada para candidatos aos cursos diurnos.

Concluindo, parece haver evidência de que os cursos noturnos tendem a favorecer o ingresso de candidatos que apresentam FSE mais baixo e que as escolas públicas federais, se comparadas às escolas públicas estaduais e municipais, estão preparando o candidato mais adequadamente para o vestibular da UFMG.

\section{ANÁLISE DO FATOR SOCIOECONÔMICO MÉDIO}

Uma vez que o FSE é uma variável fortemente associada à aprovação do candidato, cabe fazer uma análise dos cursos considerando o FSE médio de cada um. Considerando a totalidade dos candidatos percebemos que o FSE médio é de 4,66 e esse índice aumenta para 5,97, se consideramos apenas os candidatos aprovados. Analisando os candidatos aos cursos diurnos e noturnos, observamos também um aumento no FSE médio para os candidatos aprovados (de 4,98 para 6,3I para os cursos diurnos e de 3, I 5 para 4,64 para os 
Talita F. S. Dias, Ludmila V. Lage, Raphael L. F. Ribeiro, Gustavo H. M. A. Rocha, Junio G. Rodrigues, Thiago R. Santos, Glaura C. Franco, Rosangela H. Loschi e Mauro M. Braga

cursos noturnos), sendo que os candidatos aos cursos noturnos, em média, apresentam FSE mais baixo.

A figura I apresenta o FSE médio por área do curso para os candidatos inscritos e aprovados no vestibular. Percebemos que, se comparados aos cursos de outras áreas, os candidatos aos cursos de Ciências Exatas e da Terra, Ciências Humanas e Línguas, Letras e Artes são os que apresentam menor FSE médio, seja para a totalidade dos candidatos, seja para os candidatos aprovados. Percebemos também que, entre os candidatos aprovados, os que apresentam menor fator socioeconômico médio são os candidatos dos cursos de Ciências Exatas e da Terra (FSE médio $=4,9$ I) e os que apresentam maior valor para o FSE médio são os candidatos aos cursos de Ciências Agrárias (FSE médio $=6,55)$.

A tabela 5 apresenta o FSE médio de cada um dos cursos para os candidatos inscritos e os candidatos aprovados no vestibular. Se considerarmos a totalidade dos candidatos, percebemos que dos 59 cursos ofertados pela UFMG, os cursos noturnos são aqueles em que o FSE médio está entre os mais baixos. Isso indica que os cursos noturnos estão atraindo candidatos com situação socioeconômica inferior.

FIGURA I

FATOR SOCIOECONÔMICO MÉDIO POR ÁREA DO CURSO

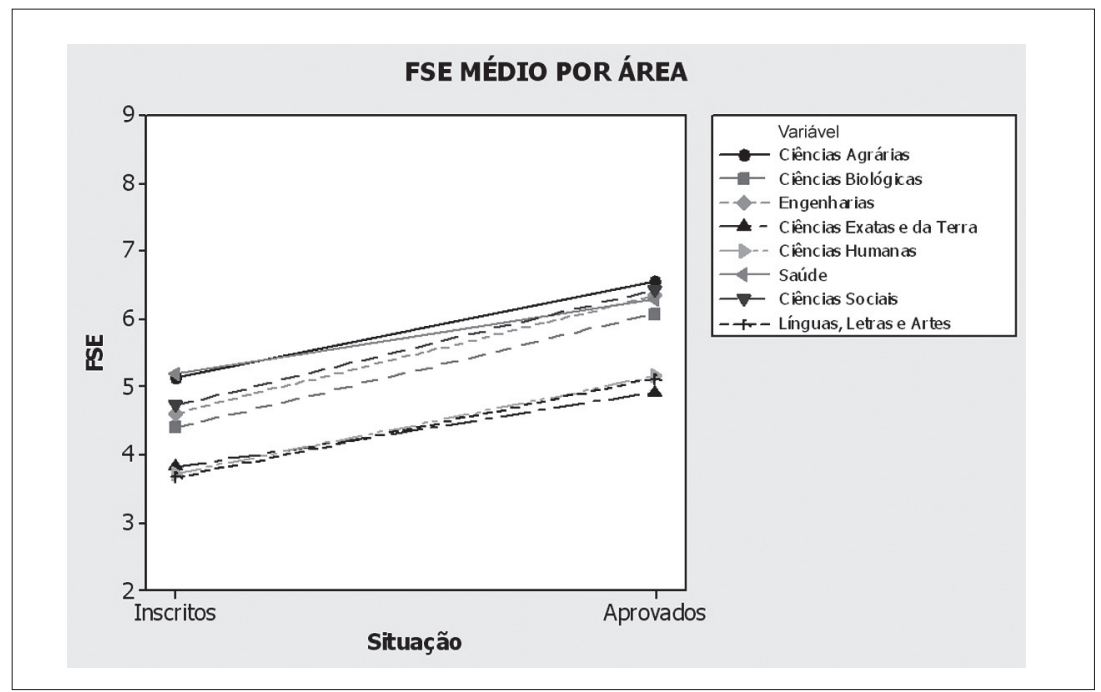


TABELA 5

FSE MÉDIO PARA A TOTALIDADE DOS CANDIDATOS

E PARA OS CANDIDATOS APROVADOS

\begin{tabular}{|c|c|c|c|c|}
\hline Área & Curso & Inscritos & Aprovados & Diferença/\% \\
\hline \multirow[t]{2}{*}{ Ciências Agrárias } & Agronomia & 4,55 & 6,19 & 36 \\
\hline & Medicina Veterinária & 5,28 & 6,66 & 26 \\
\hline \multirow[t]{2}{*}{ Ciências Biológicas } & Ciências Biológ. Noturno (Lic.) & 3,17 & 4,66 & 47 \\
\hline & Ciências Biológicas Diurno & 5,02 & 7,47 & 49 \\
\hline \multirow[t]{9}{*}{ Engenharias } & Engenharia Mecânica Noturno & 3,00 & 4,71 & 57 \\
\hline & Engenharia Metalúrgica & 3,50 & 4,90 & 40 \\
\hline & Engenharia de Minas & 4,15 & 5,18 & 25 \\
\hline & Engenharia Civil & 4,34 & 6,15 & 42 \\
\hline & Engenharia Elétrica & 4,65 & 6,57 & 41 \\
\hline & Engenharia Química & 5,26 & 6,72 & 28 \\
\hline & Engenharia. de Controle e Automação & 5,45 & 6,90 & 27 \\
\hline & Engenharia Mecânica Diurno & 5,59 & 7,77 & 39 \\
\hline & Engenharia de Produção & 5,78 & 7,53 & 30 \\
\hline \multirow[t]{7}{*}{ Ciências Humanas } & Pedagogia Noturno & 2,08 & 3,16 & 52 \\
\hline & Pedagogia Matutino & 3,04 & 4,03 & 33 \\
\hline & História Noturno (lic.) & 2,69 & 3,67 & 36 \\
\hline & Psicologia & 4,21 & 5,83 & 38 \\
\hline & História Diurno & 4,27 & 6,71 & 57 \\
\hline & Filosofia & 4,38 & 5,92 & 35 \\
\hline & Ciências Sociais & 4,44 & 6,17 & 39 \\
\hline \multirow[t]{10}{*}{ Ciências Exatas e da Terra } & Química Noturno (lic.) & 2,28 & 2,40 & 5 \\
\hline & Matemática Noturno (lic.) & 2,37 & 3,18 & 34 \\
\hline & Física Noturno (lic.) & 2,63 & 3,43 & 30 \\
\hline & Estatística & 3,21 & 4,53 & 41 \\
\hline & Matemática Diurno & 3,29 & 4,65 & 41 \\
\hline & Química Diurno & 3,70 & 5,19 & 40 \\
\hline & Matemática Computacional & 3,73 & 4,83 & 29 \\
\hline & Ciências Atuariais & 4,26 & 6,32 & 48 \\
\hline & Física Diurno (bach.) & 4,42 & 6,65 & 50 \\
\hline & Geologia & 4,46 & 5,30 & 19 \\
\hline \multirow[t]{3}{*}{ Ciências Sociais Aplicadas } & Ciência da Computação & 4,66 & 6,12 & 31 \\
\hline & Geografia Noturno (lic.) & 2,53 & 3,85 & 52 \\
\hline & Ciências Contábeis Noturno & 2,77 & 5,10 & $\begin{array}{c}84 \\
\text { (continua) }\end{array}$ \\
\hline
\end{tabular}


Talita F. S. Dias, Ludmila V. Lage, Raphael L. F. Ribeiro, Gustavo H. M. A. Rocha, Junio G. Rodrigues, Thiago R. Santos, Glaura C. Franco, Rosangela H. Loschi e Mauro M. Braga (continuação)

\begin{tabular}{|c|c|c|c|c|}
\hline \multirow{2}{*}{$\begin{array}{l}\text { Área } \\
\text { Ciências Sociais Aplicadas }\end{array}$} & \multirow{2}{*}{$\begin{array}{l}\text { Curso } \\
\text { Biblioteconomia Noturno }\end{array}$} & \multirow{2}{*}{$\begin{array}{c}\text { Inscritos } \\
1,98\end{array}$} & \multicolumn{2}{|c|}{ Aprovados Diferença/\% } \\
\hline & & & 2,78 & 40 \\
\hline & Biblioteconomia Diurno & 2,80 & 3,73 & 33 \\
\hline & Administração Noturno & 3,46 & 6,25 & 81 \\
\hline & Geografia Diurno & 4,17 & 5,61 & 35 \\
\hline & Direito Noturno & 4,20 & 6,48 & 54 \\
\hline & Turismo & 4,65 & 5,73 & 23 \\
\hline & Comunicação Social & 5,28 & 7,29 & 38 \\
\hline & Ciências Econômicas & 5,37 & 7,32 & 36 \\
\hline & Arquitetura e Urbanismo & 5,56 & 7,04 & 27 \\
\hline & Administração Diurno & 5,76 & 7,07 & 23 \\
\hline & Direito Diurno & 6,01 & 8,10 & 35 \\
\hline \multirow[t]{6}{*}{ Línguas, Letras, Artes } & Letras Noturno & 2,74 & 4,19 & 53 \\
\hline & Letras Diurno & 4,01 & 5,40 & 35 \\
\hline & Música (lic.) & 4,21 & 4,88 & 16 \\
\hline & Artes Cênicas & 4,24 & 5,57 & 31 \\
\hline & Belas-Artes & 4,45 & 6,00 & 35 \\
\hline & Música (bach.) & 4,50 & 5,97 & 33 \\
\hline \multirow[t]{9}{*}{ Ciências da Saúde } & Enfermagem & 3,81 & 5,00 & 31 \\
\hline & Fonoaudiologia & 4,03 & 5,08 & 26 \\
\hline & Educação Física & 4,20 & 5,76 & 37 \\
\hline & Terapia Ocupacional & 4,23 & 5,04 & 19 \\
\hline & Ciência da Nutrição & 4,44 & 5,75 & 30 \\
\hline & Farmácia & 4,59 & 5,30 & 15 \\
\hline & Fisioterapia & 4,85 & 6,59 & 36 \\
\hline & Odontologia & 5,06 & 6,69 & 32 \\
\hline & Medicina & 6,35 & 7,56 & 19 \\
\hline \multirow[t]{3}{*}{ Conjunto das Áreas } & Cursos diurnos & 4,98 & 6,31 & 27 \\
\hline & Cursos noturnos & 3,15 & 4,64 & 47 \\
\hline & Todos os cursos & 4,66 & 5,97 & 28 \\
\hline
\end{tabular}

Obs.: Em negrito estão destacados os cursos noturnos

Percebemos também que, para todos os cursos, há um aumento no FSE médio entre os aprovados, em relação aos inscritos. Os cursos noturnos, com exceção de Administração e Ciências Contábeis, ainda estão entre os que apresentam menor FSE médio entre os aprovados. 
Cursos diurnos e noturnos...

Embora tenhamos visto que a raça/cor não está tão fortemente associada à aprovação dos candidatos quanto, por exemplo, o tipo de escola do ensino médio, a situação com respeito à língua estrangeira e a renda mensal do grupo familiar, existe uma associação significativa dessa variável com aprovação. Se observarmos a tabela 3, verificamos que a proporção de candidatos da raça/ cor branca aumenta entre os aprovados, enquanto para todas as outras raças essa proporção diminui. Uma outra conclusão é que a proporção de candidatos inscritos e aprovados da raça/cor preta e parda é maior para os cursos noturnos.

Analisando o fator socioeconômico por raça/cor percebemos, na figura 2, que os candidatos da raça/cor preta são os que possuem fator socioeconômico médio mais baixo. Isso pode ser um indicativo de que as cotas raciais podem também cumprir o papel de realizar maior inclusão social, como pretendido pelo governo. Podemos notar ainda que candidatos das raça/cor amarela e parda têm FSE médios muito parecidos, tanto para os inscritos quanto para os aprovados. Candidatos de raça/cor branca são os que apresentam maior FSE médio.

FIGURA 2

FATOR SOCIOECONÔMICO MÉDIO POR RAÇA/COR DECLARADA

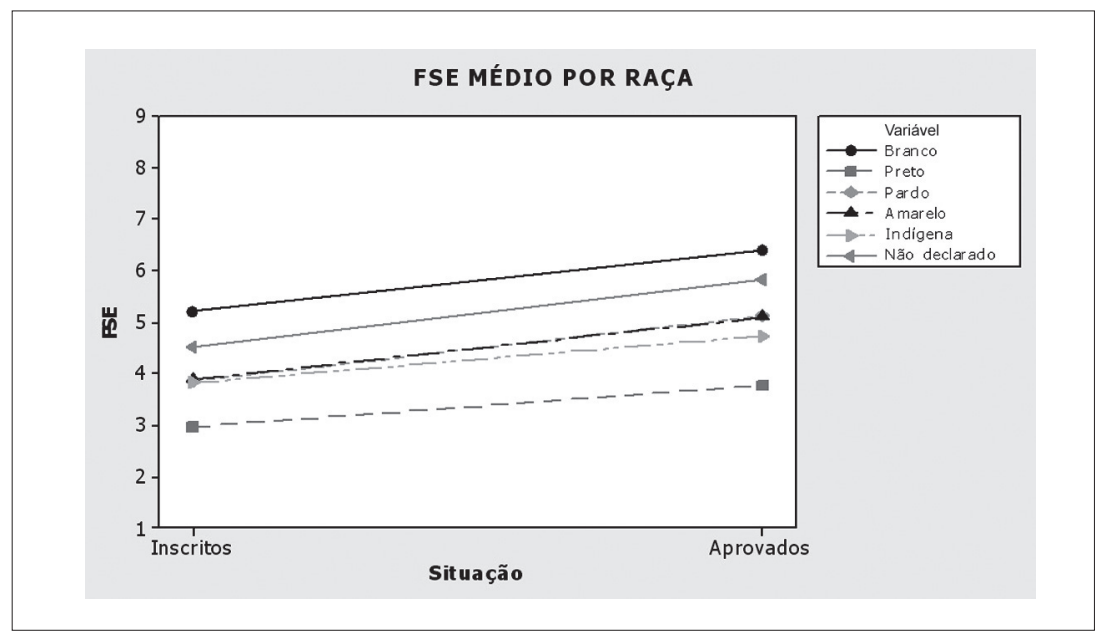


Talita F. S. Dias, Ludmila V. Lage, Raphael L. F. Ribeiro, Gustavo H. M. A. Rocha, Junio G. Rodrigues, Thiago R. Santos, Glaura C. Franco, Rosangela H. Loschi e Mauro M. Braga

\section{CONCLUSÕES}

Neste trabalho, identificamos os fatores que levaram à aprovação dos candidatos ao vestibular 2004 da UFMG, utilizando o Cart. Para a análise consideramos a totalidade dos candidatos inscritos no vestibular e também o total de candidatos inscritos para os cursos noturnos e diurnos. Também comparamos os diferentes cursos, áreas e raça/cor segundo o fator socioeconômico médio.

Os resultados do Cart mostram que a variável mais associada à variável resposta nas árvores feitas para os candidatos em geral, os candidatos aos cursos diurnos e os candidatos aos cursos noturnos, foi "situação com respeito à língua estrangeira". Notamos que a variável "classificação Abipeme" aparece somente uma vez na árvore obtida para os candidatos aos cursos noturnos, enquanto a variável FSE aparece em todas as árvores, com exceção da árvore para o diurno, podendo evidenciar que o FSE consegue explicar mais a aprovação que a classificação Abipeme. Os candidatos aos cursos noturnos apresentam comportamento um pouco diferente dos demais. Nesse grupo, algumas variáveis que não apareceram nas outras análises - tais como, classificação Abipeme, número de pessoas que vivem da renda familiar e estado civil - estão associadas à aprovação do candidato. Em relação à análise de acordo com a área do curso pretendido, podemos dizer que a variável mais associada à aprovação é o FSE, com exceção das áreas de Ciências da Saúde, Ciências Exatas e Ciências Agrárias. A variável raça/cor não se mostrou importante para explicar a aprovação do candidato em nenhum dos grupos analisados.

Em todas as análises realizadas, percebemos que o fator socioeconômico está fortemente relacionado à aprovação no vestibular. Se considerarmos a totalidade dos candidatos, percebemos que dos 59 cursos ofertados pela UFMG, os cursos noturnos são aqueles em que o FSE médio é mais baixo. Notamos também que os candidatos da raça/cor preta são os que apresentam FSE médio mais baixo e os da raça/cor branca são os que apresentam FSE médio mais alto, seja para a totalidade dos candidatos, seja apenas para os candidatos aprovados. Além disto, observamos que o FSE médio aumenta para os candidatos aprovados.

Dessa forma, concluímos que os cursos noturnos parecem estar cumprindo o papel de atrair candidatos com nível socioeconômico mais baixo, se 
Cursos diurnos e noturnos...

comparados aos cursos diurnos, apesar de o ingresso na universidade ser, em geral, mais provável para candidatos com melhor nível socioeconômico. As escolas particulares e públicas federais estão geralmente associadas à alta chance de aprovação do candidato indicando que, ao se estabelecer cotas para candidatos que concluíram o ensino médio integralmente na rede pública, pode-se favorecer um grupo de pessoas que já concorrem em pé de igualdade com candidatos que fizeram o ensino médio integralmente em escolas particulares. A baixa probabilidade de aprovação apresentada pelas escolas públicas estaduais e municipais pode ser um indicativo de que estas não estão preparando adequadamente os candidatos para o concurso vestibular, uma vez que os candidatos provenientes da rede pública federal têm, em geral, FSE tão baixo quanto os candidatos provenientes dessas escolas. A análise dos nós terminais com probabilidade de aprovação mais alta e mais baixa nas árvores construídas via Cart corroboram esta análise.

Se considerarmos apenas o FSE médio, uma vez que o fator socioeconômico é um dos principais fatores que levam à aprovação do candidato, as cotas raciais estariam cumprindo o papel de maior inclusão social já que os candidatos da raça/cor preta apresentam, em geral, menor fator socioeconômico. No entanto, tais cotas podem favorecer os candidatos da raça/cor parda, se comparados aos candidatos da raça/cor amarela e indígena, embora estes últimos grupos apresentem fator socioeconômico médio menor ou igual ao encontrado para a raça/cor parda.

\section{REFERÊNCIAS BIBLIOGRÁFICAS}

ARAÚjO, A. E. A. et al. Cursos noturnos: uma alternativa para a inclusão social no ensino superior brasileiro (estudo de caso da UFMG). In: PEIXOTO, M. C. L. (org.) Universidade e democracia: experiências e alternativas para a ampliação do acesso à universidade pública brasileira. Belo Horizonte: Editora UFMG, 2004. p. I73-187.

AZEVEDO, C. M. M. Cota racial e estado: abolição do racismo ou direitos de raça? Cadernos de Pesquisa, v.34, n.121, p.213-239, jan./abr. 2004.

BELL, J. F. Application of classification trees to the habit preference of upland birds. Journal of Applied Statistics, v.23, n.2/3, p.349-359, 1996.

BRAGA, M. M.; PEIXOTO, M.C. L.; BOGUTCHI, T. F. Tendências da demanda pelo ensino superior: estudo de caso da UFMG. Cadernos de Pesquisa, n. I I3, p. I29- I 52, jul. 200 I. 
Talita F. S. Dias, Ludmila V. Lage, Raphael L. F. Ribeiro, Gustavo H. M. A. Rocha, Junio G. Rodrigues, Thiago R. Santos, Glaura C. Franco, Rosangela H. Loschi e Mauro M. Braga

DINIZ, C. A. R.; LOUZADA-NETO, F. Data mining: uma introdução. São Paulo: Associação Brasileira de Estatística, 2000. (Simpósio Nacional de Probabilidade e Estatística, 14)

GOMES, N. L. Cotas para a população negra e a democratização da universidadede pública. In: PEIXOTO, M. C. L. (org.) Universidade e democracia: experiências e alternativas para a ampliação do acesso à universidade pública. Belo Horizonte: UFMG, 2004. p.45-56.

GUIMARÃES, A. S. Acesso de negros às universidades públicas. Cadernos de Pesquisa, n. I I8, p.247-268, mar. 2003.

LAGE, L. V. et al. Fatores que influenciaram na aprovação de candidatos de diferentes grupos socioeconômicos no vestibular-2004 da UFMG. Revista Brasileira de Estatística, v. 67, n.226, p.35-63, 2007.

LOPES, C. B. et al. Identificando as características associadas com a aprovação de candidatos de escolas públicas e privadas, vestibular-2004, UFMG. Educação em Revista, n. 46, dez. 2007.

MAGGIE, Y.; FRY, P. A Reserva de vagas para negros nas universidades brasileiras. Estudos Avançados, v. I8, n.50, p.67-80, 2004.

PEIXOTO, M. C. L.; BRAGA, M. M. Demanda pelo ensino superior no Brasil: o caso da UFMG. Educação e Linguagem, v.7, n. I 0, p. I24- 149, 2004.

PEREIRA, A. M. Um raio em céu azul: reflexões sobre a política de cotas e a identidade nacional brasileira. Estudos Afro-Asiáticos, v.25, n.3, p. 463-482, 2003.

RODRIGUES et al. Análise via Cart dos dados do vestibular 2004 da UFMG. Belo Horizonte: Projeto Vestibular UFMG, jan. 2005. (Relatório)

Recebido em: dezembro 2005

Aprovado para publicação em: julho 2007 
Cursos diurnos e noturnos...

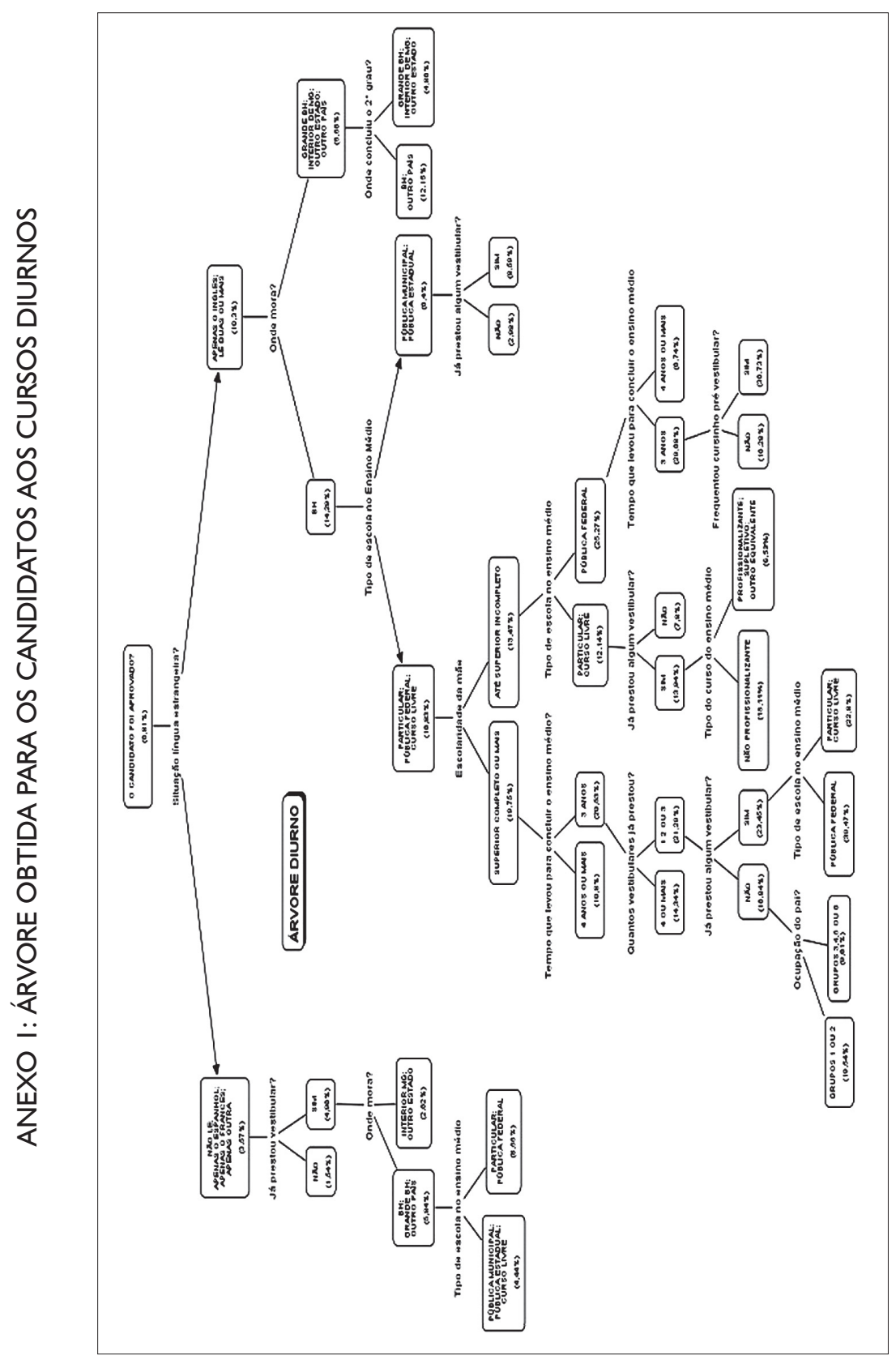

Cadernos de Pesquisa, v. 38, n. 133, jan./abr. 2008 
Talita F. S. Dias, Ludmila V. Lage, Raphael L. F. Ribeiro, Gustavo H. M. A. Rocha, Junio G. Rodrigues, Thiago R. Santos, Glaura C. Franco, Rosangela H. Loschi e Mauro M. Braga

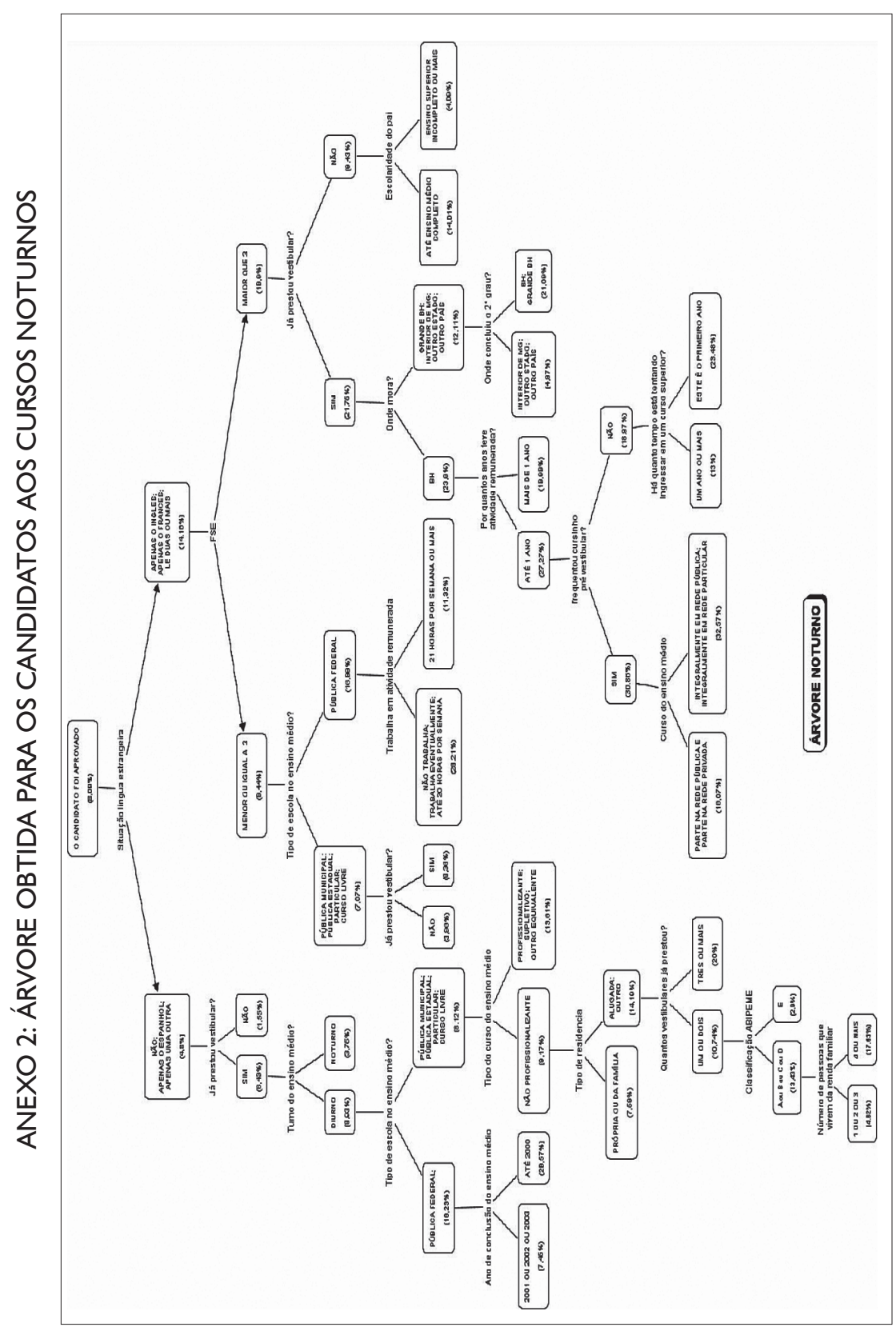

